\title{
Análisis perceptual del mercado energético basado en biomasa lignocelulósica de origen arbóreo en Costa Rica
}

\author{
Perceptual analysis of energy market based on woody biomass \\ of trees originated in Costa Rica
}

\author{
Juan Carlos Valverde ${ }^{1,2 *}$, Dagoberto Arias², Rooel Campos³, María Fernanda Jiménez ${ }^{3}$ y Laura Brenes³
}

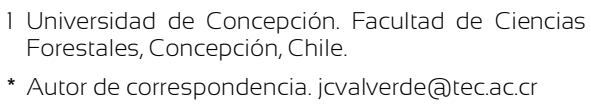

* Autor de correspondencia. jcvalverde@tec.ac.cr

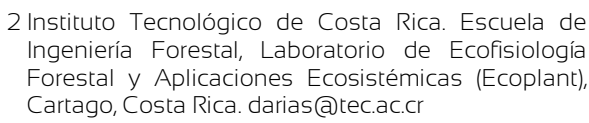

2 Instituto Tecnológico de Costa Rica. Escuela de Ingeniería Forestal, Laboratorio de Ecofisiología Forestal y Aplicaciones Ecosistémicas (Ecoplant), Cartago, Costa Rica. darias@tec.ac.cr

\begin{abstract}
3 Instituto Tecnológico de Costa Rica. Escuela de Ingeniería en Agronegocios. Cartago, Costa Rica. rocampos@tec.ac.cr, maria.jimenez@tec.ac.cr, Ibrenes@tec.ac.cr
\end{abstract}

\section{RESUMEN}

En la última década, la biomasa se ha convertido en una opción de energía renovable; sin embargo, la ausencia de estudios que permitan entender su mercado limita su implementación. El presente estudio analizó la percepción de los actores que componen el mercado de la biomasa vegetal con fines energéticos desde las perspectivas de productores, intermediarios y consumidores. Se encuestaron 52 productores, 33 intermediarios y 55 demandantes de biomasa arbórea de la zona norte de Costa Rica, estudiando el mercado desde la perspectiva técnica, ambiental, financiera y social e identificando las variables que limitan el mercado. Los resultados mostraron que para los aspectos ambientales y sociales hay una similitud de percepción entre los tres sectores; sin embargo, en los aspectos financieros y técnicos existen diferencias significativas en las percepciones, específicamente en el precio de la biomasa, en la homogeneidad de esta y en los costos asociados, además de la competencia con otras fuentes de biomasa agroindustrial. Los análisis mostraron que la variabilidad de la biomasa en cuanto a presentación afecta en $40,5 \%$ del mercado, la variación de especies en $24,8 \%$, el contenido de humedad en $10,6 \%$, la disponibilidad de biomasa en el tiempo en $8,4 \%$, la organización y estructuración del mercado en $8,9 \%$ y otras variables suman el restante $6,8 \%$. Esto hace necesaria una organización en cuanto a la venta de la biomasa y la correspondiente articulación entre ofertantes y demandantes con la participación de los intermediarios para la viabilidad del mercado de biomasa en Costa Rica.

PALABRAS ClAVE: oferta de mercado, demanda de mercado, dendroenergía, energías renovables.

\section{ABSTRACT}

In the last decade, biomass has become a renewable energy option; however, the absence of studies to understand its market limits its implementation. Therefore, the present study analyzed the perception of the actors that make up the vegetable biomass market for energy purposes from the perspectives of producers, intermediaries and consumers. For which 52 producers, 33 intermediaries and 55 consumers of tree biomass from the North Zone of Costa Rica were surveyed, studying the market from a technical, environmental, financial and social perspective, and identifying the variables that limit the market. The results showed that, for environmental and social aspects, there is a similarity of perception between the three sectors; however, in the financial and technical aspects there are significant differences, specifically in the price of biomass, homogeneity of biomass and the associated costs in addition to the competition from other sources of agro-industrial biomass. The analysis showed that biomass variability in terms of presentation affects $40.5 \%$ of the market, species variation $24.8 \%$, moisture content $10.6 \%$, biomass availability over time $8.4 \%$. Finally, the organization and structuring of the market by $8.9 \%$ and other variables add up to the remaining $6.8 \%$, being necessary an organization regarding the sale of biomass and the corresponding articulation between the sellers and consumers with the participation of intermediaries for the viability of the biomass market in Costa Rica.

KEYWORDS: market supply, market demand, dendroenergy, renewable energy. 


\section{INTRODUCCIÓN}

En la última década, a escala mundial, la demanda energética ha mostrado un incremento de 3,9\% (Lee y Yang, 2019; Specht y Madlener, 2019), debido al aumento demográfico, la automatización y la modernización de los sistemas productivos y al incremento en la cantidad de países asiáticos y latinoamericanos en vías de crecimiento económico (Gadaleta, Pellicciari y Berselli, 2019; Beluli, 2019). La presión energética ha generado la inestabilidad en los precios de venta de los combustibles fósiles, gas natural y carbón mineral, fuentes que dominan 74\% de los sistemas de generación energética mundial (Royston, Selby y Shove, 2018; Lee y Yang, 2019). Esa presión ha generado que los precios de venta de electricidad sean altos en algunos países (afectando directamente en la competitividad industrial de los países), además del consecuente incremento de las emisiones de gases de efecto invernadero, que inciden en la calidad del aire y en el fenómeno del cambio climático (Fortini y Dye, 2017; Ssempiira et al., 2018; Verlie, 2019).

Ante tal panorama, los países asiáticos y europeos han desarrollado paquetes de estímulos económicos y políticos que incentiven la investigación y desarrollo de energías renovables de bajo impacto al ambiente (Bulut y Muratoglu, 2019), que se caractericen por ser sustentables en el tiempo, financieramente competitivas y con un bajo impacto ambiental (Alimi, Rhif, \& Rebai, 2017). Cosentino et al. (2012) mencionan que, entre las tecnologías que han mostrado la mayor viabilidad y pueden ser replicadas en el mediano plazo, se encuentran la energía solar, la hidráulica, la eólica y la biomasa vegetal; esta última fuente se caracteriza por provenir de cultivos agrícolas o forestales (definida como acumulación de material orgánico por proceso de fotosíntesis en un organismo), ya sea mediante cultivos energéticos, establecidos para producir biomasa o mediante el uso y transformación de los residuos remanentes de actividades agroindustriales (Farhar, 1998). La biomasa vegetal tiene la ventaja de ser un sistema que tiene un costo financiero bajo, las emisiones de carbono que se generan por el sistema son nuevamente absorbidas por el cultivo y es de fácil producción (Popescu, Mieila, Nica y Andrei, 2018).

La potencialidad del establecimiento de la biomasa vegetal como fuente energética ha propiciado que una gran cantidad de países adopten políticas de estímulo para el desarrollo y uso de esa fuente energética (Baul, Datta y Alam, 2018; Hodges et al., 2019; Tiwary, Spasova y Williams, 2019). Cho y Kim (2019) mencionan que, para el 2050, 12,8\% de la energía consumida a escala mundial procederá de biomasa (específicamente biomasa arborea de tipo leñoso) con una tasa de crecimiento anual de 1,64\%. También se calcula que el mercado mundial bioenergético hará uso de $42 \%$ de la biomasa vegetal procedente de plantaciones forestales (Manolis, Zagas, Karetsos y Poravou, 2019), esto debido al alto poder calórico de la madera, a la capacidad de adaptación de las especies y al desarrollo tecnológico de las plantaciones de alta densidad, con sistemas de manejo intensivo y sistemas de corta sistemática. Por su parte, Zheng et al. (2018) destacan que los mercados energéticos han mostrado una tendencia hacia la implementación de cultivos de especies con alto valor calórico (Eucalyptus deglupta $4200 \mathrm{kcal} \mathrm{kg}^{-1}$, Eucalyptus grandis $4467 \mathrm{kcal} \mathrm{kg}^{-1}$ y Acacia mearnsii $4500 \mathrm{kcal} \mathrm{kg}^{-1}$ ), con un crecimiento rápido y con facilidad de homogeneizar el tamaño de la partícula, el contenido de humedad y el volumen de presentación de la biomasa consumida para el mercado demandante.

En el caso de Costa Rica, el plan de diversificación de la matriz energética del 2015 ha establecido que la biomasa forestal es una de las fuentes con mayor potencial en el país (Intituto Costarricense de Electricidad [ICE], 2015), sin embargo, carece de un mercado de oferta-demanda formal en el que se cuente con un sistema estructurado, con especificaciones claras del tipo de biomasa requerida por los demandantes y de la uniformidad del sistema de producción. Viviescas et al. (2019) destacan que el mercado oferente de biomasa en América Latina carece de precios competitivos de venta de biomasa debido a la poca homogeneidad de los residuos, entre otras cosas. El 
disponer de mercados energéticos estables y claros permite el aumento de las inversiones y el uso de la energía en otros sistemas productivos, generando con ello un sistema sustentable en el tiempo (Koengkan, Fuinhas y Marques, 2019).

Entre las técnicas de entendimiento y formulación de estrategias de mercado, se encuentra el desarrollo de estudios perceptuales, en los que se busca caracterizar y entender las perspectivas del mercado oferente y demandante hacia un producto (Mahidin, Mamat, Sani, Khoerunnisa y Kadarohman, 2019) y, a partir de ello, identificar los puntos de similitud y diferencia entre ambos sectores e identificar las barreras tecnológicas, sociales y de mercado que impiden que este último sea competitivo y atractivo a inversionistas (Lingcheng, Zhenning, Jiaping, Jing y Yuping, 2019; Valverde, Arias, Campos, Jiménez, \& Brenes, 2020). Chidanand, Sisodia y Gopalan (2019) destacan que con la percepción se puede entender no solo estructuralmente un mercado, sino también la psicología de los usuarios que forman parte de él; si se puede mejorar la idea o entendimiento de una tecnología o sistema productivo, entonces se puede incrementar su aceptación social, su implementación y con ello el cambio de paradigma (Gazijahani y Salehi, 2018). Sin embargo, es preciso entender que este tipo de estudios deben ser considerados como un primer abordaje para el desarrollo de proyectos en el que se da una idea de qué elementos se deben considerar y mejorar al momento de impulsar un nuevo desarrollo (Nematollahi, Hoghooghi, Rasti y Sedaghat, 2016). En Costa Rica, no se dispone un estudio que analice de forma clara la percepción y uso de la biomasa de origen arbóreo, siendo una limitante al momento de promocionar las alternativas de las tecnologías de optimización de la biomasa, cambio de tecnologías de combustión o uso de gasificación.

\section{OBJETIVOS}

El presente estudio tuvo los siguientes objetivos: (i) caracterizar el mercado actual de la biomasa arbórea desde la perspectiva de proveedores, intermediarios y consumidores; (ii) identificar fortalezas y debilidades del mercado actual de biomasa, así como sus potenciales puntos de mejora en el tiempo; (iii) identificar las variables de mayor sensibilidad que limitan el mercado de biomasa vegetal de Costa Rica.

\section{MATERIALES Y MÉTODOS}

\section{Sujetos de estudio}

El estudio desarrolló una encuesta entre junio y octubre del 2017. Se aplicó mediante la herramienta de encuesta digital de "Google forms" a 140 personas (Tabla 1), con una intensidad del muestreo de $6 \%$ de las organizaciones y empresas registradas legalmente en Costa Rica. La selección de las personas encuestadas se realizó considerando como requisito que debían de tener experiencia en el mercado de biomasa nacional (se revisó previamente que las personas contaran con experiencia comprobable en el tema). El grupo de estudio se dividió en tres subgrupos: ofertantes de biomasa (productores de residuos o productores de plantaciones con fines de generación de biomasa con fines energéticos), intermediarios (grupo que revende la biomasa o le aplica un proceso que proporciona un mayor valor agregado) y demandantes de biomasa (consumidor final de la biomasa). El estudio consideró la participación de encuestados en todo el país, pero se centró en la región norte de Costa Rica, debido a que es donde se desarrolla más de 50\% del mercado forestal. En total se encuestaron 140 personas procedentes de los siguientes sectores productivos: 52 productores, 33 intermediarios y 55 demandantes.

\section{Análisis de conocimiento del mercado}

El estudio analizó el conocimiento básico del mercado de biomasa, para lo cual se enfocó en cuatro perspectivas: técnica, ambiental, financiera y social (Fig. 1), realizando en cada una, de cinco a siete preguntas. Estas preguntas buscaban una cuantificación de la respuesta; mediante una escala numérica del 1 al 5 , en la que el valor 1 era el mínimo de conocimiento y 5 el máximo (las preguntas previamente se analizaron con la prueba de fiabilidad de Alpha de Cronbach, con un $\alpha=0,871)$. Con los valores obtenidos, se 
aplicó la ecuación 1, con la cual se pudo normalizar el conocimiento analizado en una escala porcentua1, siendo 100\% el valor máximo de conocimiento:

$$
C B(\%)=\left(\frac{V_{r}}{5}\right) \cdot 100 \%
$$

Donde:

$C B=$ conocimiento base comprendido de $0 \%$ a $100 \%$ $V_{r}=$ valor promedio dado por el usuario encuestado a cada temática con escala de 1 a 5 .

TABLA 1. Caracterización de la muestra de usuarios entrevistados que formaron parte del estudio de percepción del mercado de biomasa en Costa Rica.

\begin{tabular}{ccc}
\hline Variable & Frecuencia & Porcentaje \\
\hline & Género & \\
\hline Masculino & 86 & 61,43 \\
Femenino & 54 & 38,57 \\
\hline & Intervalo de edad (años) & 31,44 \\
\hline $35-35$ & 44 & 42,85 \\
$50-65$ & 60 & 25,71 \\
\hline & 36 & 7,86 \\
\hline Secundaria incompleta & Formación académica & 8,57 \\
Secundaria completa & 11 & 15,00 \\
Universitaria incompleta & 12 & 68,57 \\
Universitaria completa & 21 & \\
\hline
\end{tabular}

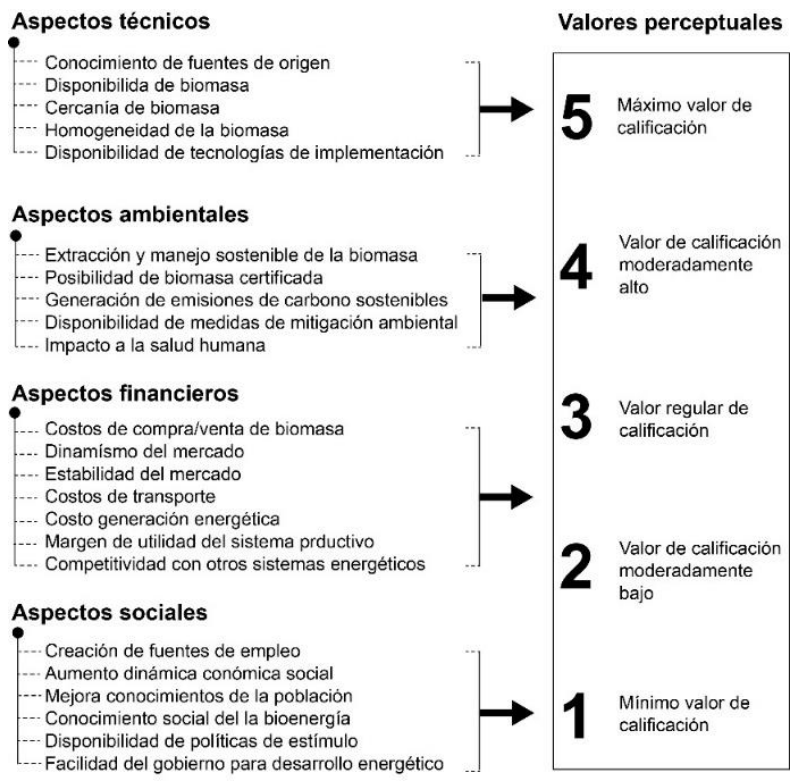

FIGURA 1. Aspectos evaluados en la caracterización de los sectores ofertante, intermediario y demandante de biomasa de origen arbóreo en Costa Rica. 


\section{Análisis de percepción}

El análisis de percepción se desarrolló mediante parejas de adjetivos opuestos, los adjetivos calificativos se seleccionaron con el fin de entender la percepción cualitativa del mercado de biomasa. Las parejas de adjetivos se colocaron intrínsecos en las preguntas durante desarrollo de la prueba formal (de tres a cuatro) para que el encuestado respondiera con una escala numérica del 1 al 5, a partir de la pareja de adjetivos. Para evitar errores por parte del grupo de estudio, previamente se realizó una prueba de fiabilidad de Alpha de Cronbach, con el fin de encontrar los grados de relación entre las preguntas y obteniendo un $\alpha=0,810$ (factor que explica una relación de las preguntas de cada temática mayor a $81 \%$ ). Las parejas de adjetivos se ordenaron con secuencia de positivo-negativo y fueron: i. Complejidad, percepción del grado de estructuración de mercado nacional (complicado-simple); ii. Estabilidad, la oferta y demanda del mercado se mantienen en el tiempo (estable-inestable); iii. Dinamismo, el mercado se encuentra en constante cambio en la oferta/demanda de biomasa (estático-dinámico); iv. Rentabilidad, el mercado genera ganancias que justifiquen la oferta/demanda de biomasa (inviable-viable); v. Organización, el mercado tiene claro el tipo de biomasa que se requiere (desorganizadoorganizado); vi. Competitividad, se tiene un mercado con una competencia significativa de compra/venta de biomasa (baja-elevada), vii. Negociabilidad, el mercado permite la negociación de precios de venta/demanda de biomasa (impermisible-permisible).

A los valores obtenidos de las parejas de objetivos se les aplicó la ecuación 1, para generar valores de 0 a 100 en donde los valores más cercanos a 0 tienen mayor relación con el adjetivo negativo (considerado como el menos funcional), mientras que los valores más cercanos a 100 tienen relación con el adjetivo positivo (considerado como el más funcional).
Aspectos de las variables que afectan el mercado y la relación de oportunidad-riesgo

Posteriormente, se analizaron las variables mediante las cuales se identificó que no hay claridad entre la percepción de ofertantes, intermediarios y demandantes de biomasa, con el fin de identificar las variables que mostraron mayor variación, entre las que están: i. Distancia de la biomasa al punto de uso (DB). ii. Costo de la fuente de energía competitiva (energía térmica) (CO). iii. Limitaciones de las políticas estatales (LE). iii. Disponibilidad tecnológica de transformación (DT). iv. Calidad de la biomasa (CA). v. Homogeneidad de la biomasa (HB). vi. Precio de la biomasa en el mercado (PB) y vii. Variabilidad de la biomasa en cuanto a las especies (VE) en el mercado de biomasa en Costa Rica.

Cada una se calificó en la escala de 1 al 5, donde el valor 1 representó el valor mínimo de "no relevante" y 5 al valor máximo considerado "relevante", posteriormente a los valores obtenidos se les aplicó la ecuación 1.

\section{Análisis estadístico}

Primeramente, se realizó un análisis descriptivo de los aspectos técnicos, ambientales, financieros y sociales de los tres segmentos para evidenciar las diferencias de cada una de las percepciones de los tres segmentos de mercado (oferente, intermediario y demandante). Para cada variable, se desarrolló la prueba de Kruskal-Wallis (previamente se realizó prueba de supuestos de normalidad) para determinar la significancia entre los sectores; a los que mostraron diferencias se les aplicó la prueba de chi-cuadrado.

En el caso de la percepción adjetiva del mercado, se realizó la prueba no paramétrica de Siegel-Tukey para comparar dos paquetes de información y para definir el grado de similitud entre los tres segmentos de mercado. Mientras que el análisis que afecta el mercado y la relación oportunidad-riesgo fue tipo SANN (Analysis/Deployment), 
a partir de la naturaleza de los datos, empleando primeramente una prueba de clasificación múltiple (log tree) para identificar las variables en las que los sectores mostraran comportamientos distintos y, posteriormente, un análisis de componentes principales para identificar los sectores con discrepancias de percepción de cada aspecto analizado.

En cuanto a los factores críticos y a los potenciales efectos de mejoras de las condiciones, se aplicó un análisis no paramétrico con la prueba de Kuiper para determinar el grado de diferenciación entre los segmentos estudiados bajo las condiciones o elementos analizados.

Finalmente, a todas las encuestas se les aplicó un análisis de Cronbach para determinar la fiabilidad y eliminar las preguntas que generaron datos erróneos o atípicos, el valor mínimo de aceptación fue de 0,80. Los análisis estadísticos se realizaron con los programas STATISTICA versión 8,9 y $\mathrm{R}$ versión 3.3 .1 con un nivel de significancia de 0,05 .

\section{Resultados}

\section{Caracterización inicial de mercado}

La caracterización inicial del mercado de biomasa desde la perspectiva de ofertantes, intermediarios y demandantes se presenta en la tabla 2. El análisis técnico determinó que en las variables: disponibilidad, tecnología, fuentes de extracción y manejo de biomasa no hay variaciones de la percepción entre los tres sectores productivos. Sin embargo, en cuanto a la cercanía de la biomasa al mercado, la homogeneidad y la disponibilidad de tecnología, se encontró una percepción diferenciada en dos agrupaciones: la primera conformada por productores e intermediarios y la otra por demandantes. Estos últimos consideraron que la cercanía de la biomasa es prioritaria, que no se cuenta con una homogeneidad de biomasa en el mercado y que el uso tecnológico es fundamental para la generación energética a partir de biomasa. Estos aspectos fueron distintos para ofertantes e intermediarios, quienes subestimaron los aspectos de distancia de las fuentes de biomasa, consideraron aceptable la heterogeneidad del mercado y también consideraron que la tecnología no es relevante.

En cuanto el aspecto ambiental, únicamente se encontraron diferencias con la variable de extracción y manejo sostenible en la biomasa, en la que los demandantes consideraron que es significativamente menor $(68,88 \%)$ en comparación con ofertantes e intermediarios, que mostraron que el manejo es mayor (promedio de 78,05\%). Para el resto de las variables, no se encontraron diferencias entre los tres segmentos de mercado; sin embargo, se encontró que la comercialización de biomasa certificada (biomasa con certificado de origen y obtenida bajo estándares de baja emisión de carbono) se nota con una posibilidad muy baja (promedio de 28,26\%). Los grupos encuestados consideraron que el uso de la bioenergía (energía producida a partir de biomasa) es un sistema de emisiones de carbono neutro y sostenible, que contribuye a la mitigación de contaminantes y no impacta la salud humana, siempre y cuando se respeten los estándares ambientales de emisiones.

El aspecto financiero fue el que mostró la mayor variación de percepción entre los tres sectores, encontrando tres tendencias de percepción que fueron: en la primera, las variables de compra/venta de biomasa y margen de utilidad del sistema, el sector demandante mostró un comportamiento significativamente diferente a ofertantes e intermediaros, considerando que el costo de venta no es el óptimo y el margen de ganancia del sistema es aceptable. La segunda agrupación fue conformada por el dinamismo y la estabilidad del mercado, aspectos en los que los demandantes e intermediarios mostraron una percepción distinta a ofertantes que consideraron que el mercado no es dinámico (no hay posibilidad de encontrar con facilidad nuevos compradores), ni estable (la venta de volumen de biomasa no es constante en el tiempo). Finalmente, la tercera agrupación no mostró diferencias entre los tres sectores, encontrando que los costos de transporte y de generación eléctrica son fundamentales para el sector, además que no hay una percepción de competir con otras fuentes energéticas disponibles en el mercado. 
TABLA 2. Caracterización porcentual inicial de los sectores ofertante, intermediario y demandante de biomasa de origen arbóreo con fines energéticos en Costa Rica.

\begin{tabular}{|c|c|c|c|}
\hline \multirow{2}{*}{ Aspecto } & \multicolumn{3}{|c|}{ Sector productivo (\%) } \\
\hline & Ofertante & Intermediarios & Demandantes \\
\hline \multicolumn{4}{|l|}{ Técnico } \\
\hline Extracción y manejo de biomasa & $77,51^{\mathrm{a}}(5,66)$ & $73,33^{\mathrm{a}}(8,44)$ & $70,05^{\mathrm{a}}(7,19)$ \\
\hline Disponibilidad de biomasa & $69,55^{\mathrm{a}}(7,07)$ & $70,01^{\mathrm{a}}(8,99)$ & $68,88^{\mathrm{a}}(9,11)$ \\
\hline Cercanía del producto al mercado & $85,88^{\mathrm{a}}(9,80)$ & $89,77^{\mathrm{a}}(8,99)$ & $95,66^{\mathrm{b}}(4,33)$ \\
\hline Homogeneidad de la biomasa & $58,11^{\mathrm{a}}(9,99)$ & $57,11^{\mathrm{a}}(7,55)$ & $30,05^{b}(8,00)$ \\
\hline Disponibilidad tecnológica & $88,12^{\mathrm{a}}(6,66)$ & $82,22^{\mathrm{a}}(7,10)$ & $89,99^{a}(6,55)$ \\
\hline \multicolumn{4}{|l|}{ Ambiental } \\
\hline Extracción y manejo sostenible de biomasa & $77,11^{\mathrm{a}}(6,13)$ & $78,99^{\mathrm{a}}(9,29)$ & $68,88^{\mathrm{b}}(11,22)$ \\
\hline Posibilidad de biomasa certificada & $27,11^{\mathrm{a}}(6,13)$ & $28,79^{\mathrm{a}}(9,29)$ & $28,88^{\mathrm{a}}(11,22)$ \\
\hline Generación de emisiones de carbono sostenibles & $90,33^{\mathrm{a}}(4,44)$ & $91,22^{\mathrm{a}}(5,00)$ & $89,11^{\mathrm{a}}(5,16)$ \\
\hline Disponibilidad de medidas de mitigación & $88,66^{\mathrm{a}}(8,33)$ & $86,61^{a}(7,11)$ & $84,12^{\mathrm{a}}(6,11)$ \\
\hline Impacto a la salud humana & $90,90^{a}(5,66)$ & $92,11^{\mathrm{a}}(6,01)$ & $94,00^{\mathrm{a}}(5,14)$ \\
\hline \multicolumn{4}{|l|}{ Financiero } \\
\hline Costos de compra/venta de biomasa & $34,11^{\mathrm{a}}(4,89)$ & $55,11^{\mathrm{b}}(6,33)$ & $30,11^{\mathrm{a}}(3,00)$ \\
\hline Dinamismo de mercado (Compra/venta) & $56,99^{\mathrm{a}}(5,55)$ & $75,44^{\mathrm{b}}(7,70)$ & $69,55^{\mathrm{b}}(8,90)$ \\
\hline Estabilidad del mercado & $84,00^{a}(6,00)$ & $68,65^{\mathrm{b}}(5,09)$ & $66,53^{\mathrm{b}}(7,88)$ \\
\hline Costos de transporte & $82,77^{\mathrm{a}}(3,58)$ & $81,39^{a}(5,60)$ & $86,33^{\mathrm{a}}(5,11)$ \\
\hline Costo de generación eléctrica & $74, \mathrm{Ol}^{\mathrm{a}}(5,22)$ & $72,23^{\mathrm{a}}(8,13)$ & $70,99^{a}(6,71)$ \\
\hline Margen de utilidad del sistema productivo & $44,29^{\mathrm{a}}(5,99)$ & $44,50^{a}(7,39)$ & $64,89^{\mathrm{b}}(5,33)$ \\
\hline Competitividad con otros sistemas energéticos & $24,00^{a}(6,11)$ & $23,55^{\mathrm{a}}(6,22)$ & $20,01^{\mathrm{a}}(6,42)$ \\
\hline \multicolumn{4}{|l|}{ Social } \\
\hline Creación de fuentes de empleo & $88,88^{\mathrm{a}}(4,11)$ & $89,97^{\mathrm{a}}(7,22)$ & $90,15^{\mathrm{a}}(7,00)$ \\
\hline Aumento de la dinámica económica social & $60,61^{\mathrm{a}}(8,00)$ & $63,33^{\mathrm{a}}(7,98)$ & $67,77^{\mathrm{a}}(8,11)$ \\
\hline Conocimiento de la población del uso de la biomasa & $64,47^{\mathrm{a}}(5,33)$ & $50,10^{\mathrm{b}}(8,23)$ & $64,30^{a}(6,55)$ \\
\hline Disponibilidad de políticas de estímulo & $30,22^{\mathrm{a}}(6,89)$ & $26,77^{\mathrm{a}}(5,66)$ & $22,33^{\mathrm{b}}(7,22)$ \\
\hline Facilidad del gobierno para desarrollo bioenergético & $62,30^{\mathrm{a}}(4,19)$ & $60,00^{a}(7,18)$ & $53,33^{\mathrm{b}}(6,89)$ \\
\hline
\end{tabular}

Letras distintas denotan diferencias con una significancia del 0,05.

Con las variables financieras, ofertantes y demandantes mostraron que los precios de comercialización no son ideales, en comparación con los intermediarios, quienes los consideraron aceptables. La percepción significativamente menor fue presentada por los demandantes de biomasa, mientras ofertantes e intermediarios la consideraron significativamente mayor. La disponibilidad de tecnología adquirible fue percibida de igual forma por parte de los tres sectores. Finalmente, en cuanto a la perspectiva de costos de oportunidad, los tres sectores consideraron que existen en el mercado fuentes de energía alternativas competitivas (energía a partir de combustibles fósiles) con costos aceptables y con una homogeneidad mayor (especialmente notada por los demandantes). En conjunto se considera que las políticas actuales no son las mejores para el sector de biomasa.

En cuanto el aspecto social, los tres sectores productivos mostraron una buena percepción en cuanto a generación de empleo, mejora de la dinámica de la economía rural y entendimiento del uso de la biomasa 
como fuerte energética por parte de la población. Sin embargo, en aspectos de índole política (políticas de estímulo y facilidades de las instituciones del estado), es el sector demandante de biomasa el que consideró que las políticas son negativas y el desarrollo bioenergético en Costa Rica es complicado.

\section{Percepción adjetiva del mercado actual}

La percepción de mercado (Fig. 2) mostró cuatro comportamientos, según la pareja de adjetivos calificativos. Primeramente, no se encontraron diferencias entre los sectores productivos, para la complejidad del mercado, dinamismo y organización (Fig. 2a, 2c y 2e). Los tres sectores mostraron una misma percepción, en el sentido de que el mercado es complejo, con una dinámica de oferta y demanda elevada y un sistema de organización bajo. El segundo comportamiento atribuido al sector intermediario mostró una percepción distinta a la de ofertantes y demandantes (ambos no mostraron diferencias significativas); esto se presentó con la variable de estabilidad de mercado (Fig. 2b), en la que los intermediarios mostraron que la percepción del mercado es poco estable con un valor de $36,5 \%$ en comparación con ofertantes y demandantes que mostraron una percepción moderadamente estable (promedio 56,6\%). Lo anterior es debido a que el intermediario debe conectar a ofertantes $y$ demandantes; de ahí que se considere que la localización de fuentes de biomasa sea más complicada que el proceso de venta directa de los ofertantes a los demandantes de biomasa.

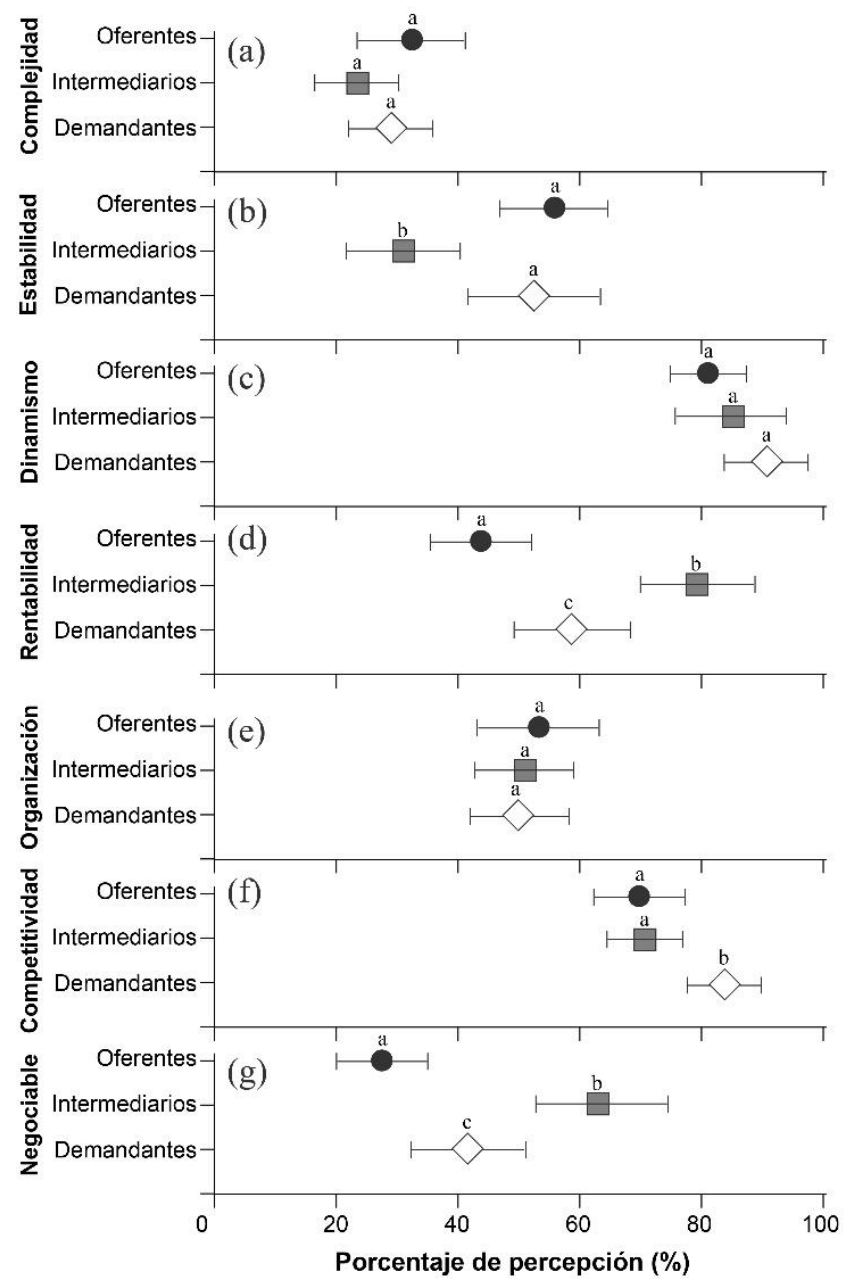

FIGURA 2. Percepción del mercado por parte de ofertantes, intermediarios y demandantes de biomasa a partir de adjetivos calificativos del mercado de biomasa de Costa Rica. 
El tercer comportamiento se dio con la variable de competitividad (Fig. 2f), en la que los demandantes de biomasa consideraron que el mercado es significativamente más competitivo $(84,6 \%)$, en comparación con ofertantes e intermediarios $(70,9 \%)$, debido a una exigencia de biomasa homogénea (en especie y presentación). Finalmente, el cuarto comportamiento, en el que los tres sectores no concordaron en criterios, fue el de las variables de rentabilidad y negociabilidad de biomasa (fig. $2 \mathrm{~d}$ y $2 \mathrm{~g}$ ). Los intermediarios consideraron que es altamente rentable $(78,5 \%)$, en contraste con los ofertantes, que consideraron que es poco rentable $(44,4 \%)$ y los demandantes que mostraron un valor medio (59,1\%). Estas variaciones son producidas por la fluctuación del margen de utilidades e inversiones necesarias en cada segmento del mercado. En cuanto a negociabilidad, los intermediarios consideraron que el mercado permite el desarrollo de negociaciones del precio de biomasa de una forma elevada $(63,7 \%)$, mientras que los ofertantes consideraron que se dificulta la capacidad de negociación y un segmento de los entrevistados la considera nula $(30,1 \%)$, debido a los bajos precios de compra por parte de los intermediarios o a los requerimientos de la biomasa por parte del mercado.

\section{Análisis de variables que afectan el mercado y relación oportunidad-riesgo}

$\mathrm{Al}$ analizar las variables que los tres sectores consideran que afectan el mercado de biomasa (Fig. 3), inicialmente se encontró que las variables distancia de la biomasa al punto de uso (DB), costo de alternativas de energía competitivas (CO), limitaciones de las políticas estatales (LE) y disponibilidad tecnológica de transformación (DT) no mostraron mayor diferenciación entre criterios de los tres sectores (Fig. 3a), lo cual se evidenció con un distanciamiento eucleriano inferior a 0,23. Al analizar la variación interna de cada variable de este grupo (fig. 3b), se encontró para DB, que la posibilidad de mejora de esta condición tiene un riesgo bajo pero una oportunidad de incidencia en el mercado potencialmente elevada. Sucede algo similar con LE, en que se menciona que un apoyo gubernamental en cuanto al uso de biomasa o la mejora de las condiciones actuales de la venta energética tendría un potencial beneficio al mercado y con un riesgo negativo. Por su parte, con DT se mantiene la percepción de que una mejora en cuanto a la localización de la biomasa para la venta mejoraría el mercado y con un riesgo negativo, lo cual incidiría positivamente en el sector. Sucede lo opuesto con $\mathrm{CO}$, una mejora de los precios de la energía implicaría un alto riesgo al mercado de biomasa y un beneficio negativo (imposibilidad de nuevas oportunidades de crecimiento).

La segunda agrupación euclidiana fue conformada por las variables calidad de la biomasa (CA) y homogeneidad de la biomasa (HB), en ambas el sector demandante de biomasa mostró un comportamiento distinto al de ofertantes e intermediarios (estos últimos mostraron el mismo comportamiento). En cuanto a CA, ofertantes e intermediarios consideran que la venta de biomasa con mayor calidad si bien puede inferir en una mejora de mercado (mayor oportunidad de negociación), tiene un riesgo moderado ya que su precio tendría que aumentar por los estándares de calidad (biomasa con mayor homogeneidad). En cambio, los demandantes consideran que la biomasa de mayor calidad tendría un riesgo mínimo, pero con un impacto muy favorable al mercado (alta oportunidad de comercialización).

La tercera agrupación euclidiana consistió en la variable precio de la biomasa en el mercado (PB), en el que se encontró una disonancia significativa entre los tres sectores, tanto ofertantes como intermediarios, consideran que una mejora en los precios de biomasa (diminución del precio de mercado) implicaría un aumento en el mercado y venta de biomasa (mayor oportunidad), pero el riesgo sería mayor (significativamente mayor para los intermediarios), debido a que los márgenes de utilidad se disminuirían y no se garantizaría continuidad de sustentar la demanda en el tiempo. En contraposición, los demandantes consideran que una mejora en los precios incidiría positivamente en la oportunidad de ampliación de uso de la biomasa y consideran que el riesgo sería negativo (no habría riesgos de la realización de la acción). Finalmente, la variabilidad de la biomasa en cuanto a especies y tipo (VE), mostró el mayor 
distanciamiento eucleriano $(9,86)$, encontrando que una mejora de dichas condiciones incidiría favorablemente en los demandantes, con riesgo negativo y alta oportunidad de aumento de la demanda (se garantizaría biomasa homogénea para los sistemas productivos). En cambio, los intermediarios consideran que, si bien la oportunidad de homogenizar la biomasa es buena, se corre un elevado riesgo por conseguir fuentes que satisfagan la demanda. Esto es similar a lo percibido por los ofertantes, que consideran que dicha acción más bien limitaría las oportunidades en el mercado con un riesgo significativo, ya que se limitaría, las especies y las condiciones de demanda, aspectos que reducirían las especies ofertadas en el mercado y los potenciales volúmenes que se puedan ofertar.

\section{Identificadores de puntos críticos de mercado e impacto de potenciales mejoras}

A partir de las percepciones de los tres segmentos del mercado en las distintas perspectivas analizadas y determinando las variables que inciden directamente en el mercado, se identificaron las variables que se consideraron como aspectos críticos en el desarrollo y futuro del uso de biomasa como fuente de energía (Tabla 3), se identificaron siete aspectos fundamentales. El primero consistió en las características de la biomasa, específicamente la variabilidad de las especies (menor cantidad de especies posible, las que cuenten con mayor área de plantación), el contenido de humedad (inferior a 20\%) y la presentación de la biomasa (en preferencia en astillas o "pellets"). En los tres criterios se presentó el mismo comportamiento, en el sentido de que el mercado demandante menciona que actualmente es inaceptable la gran variedad de especies, contenidos de humedad elevados y heterogeneidad de la biomasa disponible en el mercado. Estos aspectos implican que las tecnologías disponibles no alcancen su máxima eficiencia o se requieran procesamientos extras (secado de biomasa, transformación de la biomasa), para que sea compatible con los equipos operativos. En contraposición, los ofertantes y los demandantes consideran que la biomasa disponible es aceptable, esto se evidencia pues solo $6 \%$ de los encuestados la considera como inaceptable; identificando con ello una discrepancia entre ambos sectores.
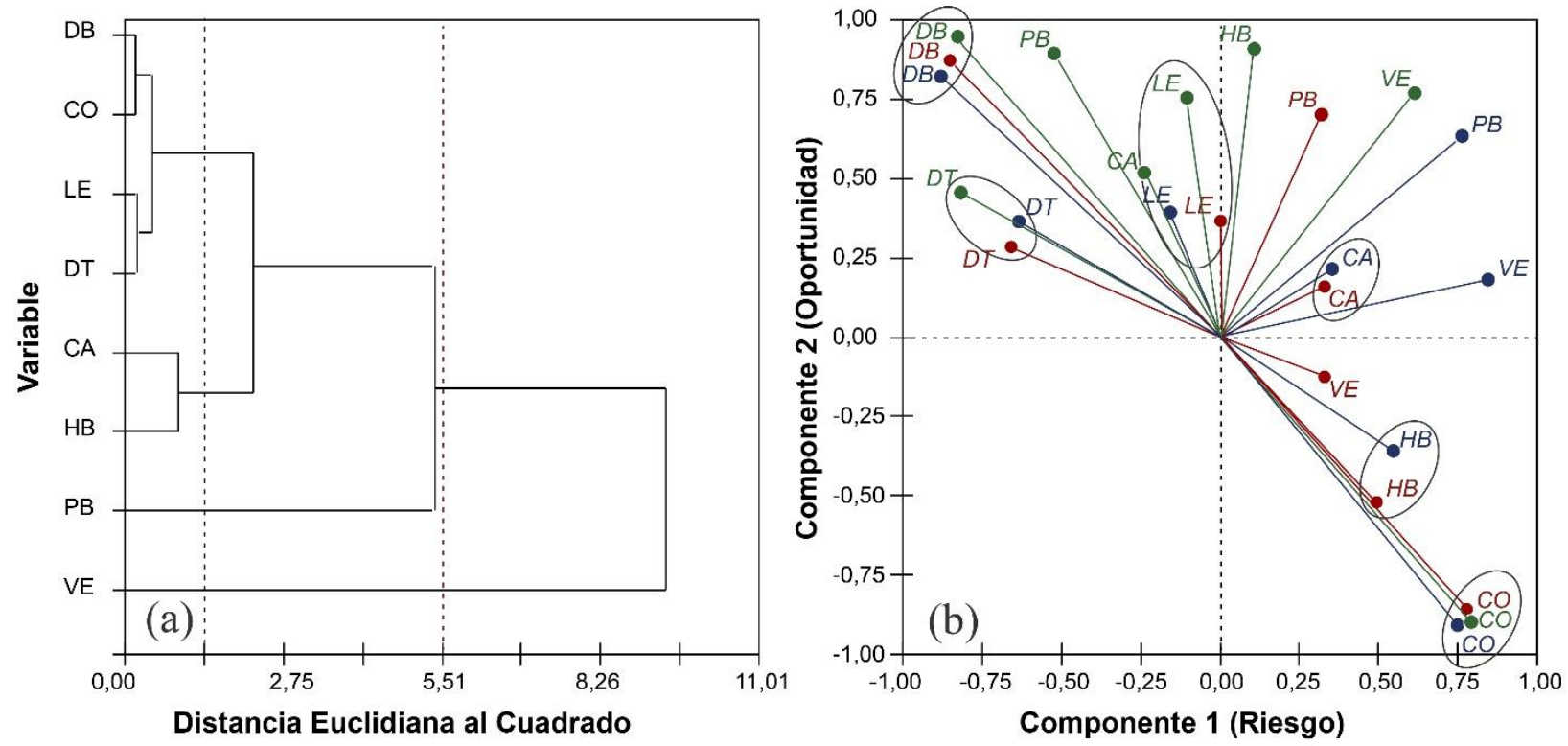

FIGURA 3. Análisis de dendrograma de conglomerados y componentes principales (función de oportunidad y riesgos del mercado) en el mercado de biomasa en Costa Rica.

Distancia de la biomasa al punto de uso (DB), costo de fuente de energía competitivas (energía térmica) (CO), limitaciones de políticas estatales (LE), disponibilidad tecnológica de transformación (DT), calidad de la biomasa (CA), homogeneidad de la biomasa (HB), precio de la biomasa en el mercado (PB), variabilidad de biomasa en cuanto especies (VE). 
TABla 3. Porcentajes de aceptabilidad de los factores críticos del mercado identificados a partir del sector oferente, intermediario y demandante de biomasa en Costa Rica

\begin{tabular}{|c|c|c|c|c|}
\hline \multirow{2}{*}{ Aspecto } & \multirow{2}{*}{ Sector productivo } & \multicolumn{3}{|c|}{ Criterio obtenido (\%) } \\
\hline & & Aceptable & Tolerable & Inaceptable \\
\hline \multirow{3}{*}{$\begin{array}{l}\text { Variación de biomasa por } \\
\text { especies }\end{array}$} & Oferente & 33,3 & $62,5^{*}$ & 4,2 \\
\hline & Intermediarios & 34,3 & $60,6^{*}$ & 5,1 \\
\hline & Demandantes & 13,4 & 30,8 & $55,8^{* *}$ \\
\hline \multirow{3}{*}{$\begin{array}{l}\text { Variación de biomasa por } \\
\text { presentación }\end{array}$} & Oferente & 30,5 & $40,4^{*}$ & 29,1 \\
\hline & Intermediarios & $30,4^{*}$ & $42,2^{*}$ & 2,4 \\
\hline & Demandantes & 15,1 & 35,9 & $49,0^{* *}$ \\
\hline \multirow{3}{*}{$\begin{array}{l}\text { Variación de biomasa por } \\
\text { contenido de humedad }\end{array}$} & Oferente & 20,5 & $50,3^{*}$ & 29,2 \\
\hline & Intermediarios & 33,9 & $45,7^{*}$ & 20,4 \\
\hline & Demandantes & 5,6 & 30,9 & $63,5^{* *}$ \\
\hline \multirow{3}{*}{$\begin{array}{l}\text { Disponibilidad de biomasa en } \\
\text { el tiempo }\end{array}$} & Oferente & 20,8 & $40,5^{*}$ & 38,7 \\
\hline & Intermediarios & 18,8 & $33,9^{*}$ & 47,3 \\
\hline & Demandantes & 20,9 & $30,9^{*}$ & 48,2 \\
\hline \multirow{3}{*}{$\begin{array}{l}\text { Organización del mercado de } \\
\text { biomasa }\end{array}$} & Oferente & 15,5 & 30,3 & $54,2^{* *}$ \\
\hline & Intermediarios & 33,9 & $55,2^{*}$ & 10,9 \\
\hline & Demandantes & 10,9 & 16,6 & $50,5^{* *}$ \\
\hline \multirow{3}{*}{$\begin{array}{l}\text { Políticas de estímulo del } \\
\text { mercado bioenergético }\end{array}$} & Oferente & 10,2 & 20,2 & $69,6^{*}$ \\
\hline & Intermediarios & 9,9 & 33,7 & $56,4^{*}$ \\
\hline & Demandantes & 5,9 & 22,2 & $71,9^{*}$ \\
\hline \multirow{3}{*}{$\begin{array}{l}\text { Disponibilidad tecnológica de } \\
\text { procesamiento biomasa }\end{array}$} & Oferente & 33,5 & $60,1^{*}$ & 6,4 \\
\hline & Intermediarios & 30,5 & $50,4^{*}$ & 19,2 \\
\hline & Demandantes & 25,5 & $49,9^{*}$ & 24,6 \\
\hline
\end{tabular}

* significancia al 0,05; ** significancia al O,Ol,

Otro aspecto crítico que se determinó fue la disponibilidad de biomasa en el mediano y largo plazo, se determinó que los demandantes consideran que serán insuficientes los volúmenes que estarán disponibles. Por otra parte, con respecto a la organización del mercado actual hay una clara discrepancia, para demandantes y ofertantes el mercado actual es inaceptable y carece de una estructura clara en la que la calidad y estabilidad de la biomasa sea manejable a largo plazo; en cambio, los intermediarios consideran que el mercado es aceptable, que permite la negociación y manejo de la biomasa con los sectores ofertante y demandante y en que el aspecto de trasformación y mejora de biomasa es fundamental para el mercado.

En cuanto a los aspectos de políticas del mercado de bioenergía y a la disponibilidad de tecnologías de procesamiento de biomasa, los tres sectores mantienen una homogeneidad de criterios; para la primera variable se considera que las políticas actuales son inaceptables, debido a que los precios fijados (los intervalos de negociación de precio son muy limitados) en el mercado imposibilitan o, en el mejor de los casos, limitan la venta de excedentes de generación eléctrica, propiciando que la mayoría de los demandantes de biomasa tiendan a modalidades de uso 
para el auto consumo, a la vez que ofertantes e intermediarios de biomasa cuentan con limitaciones en cuanto a la tramitología de los permisos necesarios para la realización de las operaciones. En el caso de los aspectos tecnológicos, se registra una percepción tolerable, pero queda clara la apreciación de que en el mediano plazo será necesaria una modernización tecnológica.

$\mathrm{Al}$ analizar las mejoras potenciales del mercado de biomasa (Tabla 3), se presentaron siete acciones, las cuales se examinaron desde el punto de vista de la posibilidad de incremento, manutención o decrecimiento del mercado en el mediano y largo plazo (Tabla 4). Al mejorar la calidad de la biomasa (creando categorías de calidad de biomasa y mejorando los sistemas de obtención y transformación de biomasa), la percepción mostró el mismo comportamiento con una expectativa de 53,6\% de los sectores en que el mercado se incrementaría, mientras $34,0 \%$ consideraría se mantendría sin variación. Esto se debe a que, al tener una materia prima más homogénea, se dispondría de una mayor adaptación a las condiciones tecnológicas nacionales. Con las variables de diminución de costos operativos y mejora de los precios de comercialización de biomasa, se mantiene la tendencia de percepción de que el mercado crecería (más de $75 \%$ lo considera), ya que se tendría un mayor margen de ganancia para los distintos sectores a la vez que se permitirían reinversiones en los procesos.

TABLA 4. Porcentaje de percepción de impacto en la mejora de debilidades identificadas el mercado de biomasa, a partir del sector oferente, intermediario y demandante en Costa Rica.

\begin{tabular}{|c|c|c|c|c|}
\hline \multirow{2}{*}{ Aspecto } & \multirow{2}{*}{ Sector productivo } & \multicolumn{3}{|c|}{ Proyección del mercado (\%) } \\
\hline & & Incremente & Mantenga & Disminuya \\
\hline \multirow{3}{*}{$\begin{array}{l}\text { Mejora de la calidad } \\
\text { de la biomasa }\end{array}$} & Oferente & $50,5^{* *}$ & 38,5 & 11,0 \\
\hline & Intermediarios & $50,5^{* *}$ & 32,5 & 17,0 \\
\hline & Demandantes & $60,9^{* *}$ & 34,9 & 4,2 \\
\hline \multirow{3}{*}{$\begin{array}{l}\text { Disminución de los } \\
\text { costos operativos de } \\
\text { la biomasa }\end{array}$} & Oferente & $70,5^{* *}$ & 25,6 & 3,9 \\
\hline & Intermediarios & $75,8^{* *}$ & 22,9 & 1,3 \\
\hline & Demandantes & $82,2^{* *}$ & 16,5 & 1,3 \\
\hline \multirow{3}{*}{$\begin{array}{l}\text { Mejora en precio de } \\
\text { comercialización de } \\
\text { biomasa }\end{array}$} & Oferente & $75,8^{* *}$ & 20,1 & 4,1 \\
\hline & Intermediarios & $80,2^{* *}$ & 15,5 & 4,3 \\
\hline & Demandantes & $82,1^{* *}$ & 12,9 & 5,0 \\
\hline \multirow{3}{*}{$\begin{array}{l}\text { Creación programa de } \\
\text { generación continua } \\
\text { de biomasa }\end{array}$} & Oferente & $77,8^{* *}$ & 20,2 & 2,0 \\
\hline & Intermediarios & $60,5^{* *}$ & 30,2 & 9,3 \\
\hline & Demandantes & $80,5^{* *}$ & 18,8 & 0,7 \\
\hline \multirow{3}{*}{$\begin{array}{l}\text { Mejora organización } \\
\text { del mercado de } \\
\text { biomasa }\end{array}$} & Oferente & $80,9^{* *}$ & 15,9 & 3,2 \\
\hline & Intermediarios & 30,9 & $40,1^{* *}$ & 29,0 \\
\hline & Demandantes & $90,1^{* *}$ & 8,0 & 1,9 \\
\hline \multirow{3}{*}{$\begin{array}{l}\text { Creación de políticas } \\
\text { de estímulo del } \\
\text { mercado de biomasa }\end{array}$} & Oferente & $89,9^{* *}$ & 9,8 & 0,3 \\
\hline & Intermediarios & $82,1^{* *}$ & 7,5 & 10,4 \\
\hline & Demandantes & $92,8^{* *}$ & 6,6 & 0,6 \\
\hline \multirow{3}{*}{$\begin{array}{l}\text { Implementación } \\
\text { nuevas tecnologías } \\
\text { para uso biomasa }\end{array}$} & Oferente & $88,8^{* *}$ & 12,0 & 0,8 \\
\hline & Intermediarios & $45,9^{*}$ & $50,1^{*}$ & 4,0 \\
\hline & Demandantes & $80,0^{* *}$ & 18,7 & 1,3 \\
\hline
\end{tabular}

Nota: * significancia al O,O5; ** significancia al O,Ol. 
Con la mejora potencial de organización del mercado, ofertantes y demandantes consideran de forma significativa que se daría un aumento del mercado; sin embargo, los intermediarios consideran que se mantendría estable; esto debido a la lejanía geográfica entre ofertantes y demandantes en conjunto con la competitividad con otras fuentes de energía. En cambio, con el aspecto de políticas que estimulen el mercado, los tres sectores consideran que mejorarían las condiciones, debido a que el mercado se volvería más atractivo y competitivo con respecto a las otras fuentes energéticas.

\section{DISCUSIÓN}

\section{Situación actual del mercado}

Los resultados mostraron que, a escala nacional, los sectores productivos muestran una concordancia en aspectos ambientales y sociales (tabla 1). Se considera que la implementación de biomasa como fuente energética incide en reducción de emisiones de carbono, aspecto que es relevante en los procesos de economía carbono neutral. Morseletto (2020) destaca que las economías a partir de la bioenergía inciden positivamente en el ambiente, ya que la materia prima del sistema secuestra emisiones de $\mathrm{CO}_{2}$, mismas que se generan en el procesos de transformación energética, incidiendo en un sistema cerrado de emisiones. Esto se complementa con lo destacado por FranklinJohnson y Canning (2016), quienes aseveran que el uso de la biomasa incide en la reactivación económica de áreas rurales, regiones en las que se dispone de áreas significativas para el desarrollo de áreas de cultivos dendroenergéticos y fuentes sostenibles de biomasa leñosa, misma que se puede incluir en programas bioenergéticos de sostenibilidad productiva. Los estudios de Cambero, Alexandre y Sowlati (2015), Simangunsong et al. (2017) y de Röder et al. (2019) mencionan que el uso de bioenergía incide tanto en la creación de nuevas fuentes energéticas competitivas para el mercado internacional, como en el conjunto de mejoras de las condiciones rurales o de poco desarrollo industrial, por lo que un mercado energético de biomasa permite la participación directa e indirecta de productores, organizaciones, comunidades, regiones poco desarrolladas y reactivación productiva de terrenos de poco uso o con subutilización del suelo.

Por otra parte, las diferencias entre las percepciones técnicas y financieras se deben a las perspectivas de cada sector productivo. Pang et al. (2017) encontró para el mercado europeo diferencias de percepción entre intermediarios, ofertantes y demandantes de biomasa y se debe al enfoque meta de cada sector. En el caso de los productores, la meta se enfoca en colocar la biomasa en el mercado, así, el volumen de comercialización pasa a ser relevante y su presentación, contenido de humedad y calidad inciden directamente en el precio; por su parte los intermediarios se enfocan en la compra de biomasa en cualquier condición de venta, con subprocesos para incrementar su valor o en su defecto optimizar el transporte de la biomasa al consumidor. El demandante se enfoca en la compra de la biomasa bajo sus requerimientos y los modelos de generación energética, ya sea para comercializar en el mercado o para autoabastecimiento. Para el caso de Costa Rica, las perspectivas son similares, pero ofertantes e intermediarios le dan un valor menor a la calidad y homogeneidad de la biomasa, ya que consideran que su posibilidad de venta se verá restringida o la posibilidad de negociación será negativa. Esta percepción concuerda con lo destacado por Kahrl, Su, Tennigkeit, Yang y Xu (2013) en el mercado energético del Norte de China; estos autores encontraron que, conforme la calidad y homogeneidad de la biomasa sea mayor, la capacidad de negociación se vuelve menor por parte de los ofertantes, ya que se reduce el margen de ganancia y se crea una dependencia a los demandantes específicos de la materia prima, incidiendo en una reducción del mercado.

Estas diferencias entre el mercado vendedor y comprador de biomasa, en conjunto con una organización reducida y con políticas de estímulo limitadas y que en muchos casos exceden las regulaciones de los procesos de generación de biomasa, ocasionan que el mercado se perciba como desordenado. En un estudio de Cho y Kim (2019), este aspecto es percibido como normal en las regiones tropicales, lo cual genera que la bioenergía con 
respecto a otras fuentes de energías como los combustibles fósiles o gas natural sea menos competitiva, ya que dichas fuentes son muy homogéneas y están en un mercado muy estructurado y competitivo. Viviescas et al. (2019) destacan que es necesaria una mejora de mercados de la biomasa en Latinoamérica que permita que la biomasa se adapte de mejor manera a los sistemas energéticos demandantes y, con ello, se reduzcan los subprocesos o entradas y otros factores de mercado que incrementan su costo. Además, se deben crear sistemas de estímulos regionales en los que la adquisición de tecnologías o el desarrollo de organizaciones bioenergéticas o clúster cuenten con una posibilidad de organizarse y competir en el mercado energético.

\section{Efectos de la disonancia entre ofertantes, demandantes e intermediarios}

Como se ha mencionado anteriormente, la heterogeneidad de la biomasa, en conjunto con los precios de comercialización (bajos márgenes de ganancia) y competencias desventajosas con respecto a otras fuentes energéticas como la térmica, propician que el mercado de la biomasa sea considerado como complejo, dinámico y competitivo; sin embargo, carece de organización y de constancia en el tiempo. Nematollahi et al. (2016) han identificado que se debe aumentar la competitividad de las energías renovables, mediante organizaciones estructuradas en las que ofertantes, demandantes e intermediarios estén debidamente inscritos y en las que las características de la materia prima ofertada/demandada sean estandarizadas. El creciente aumento de nuevas tecnologías que optimizan el potencial calórico de la biomasa trae como consecuencia la necesidad de materia prima con estándares claros en cuanto contenido de humedad, dimensiones, especie y flujo de volumen. La nueva generación de calderas y gasificadores de alta eficiencia han optado por el uso de "pellets" o astillas bajo normas internacionales, con el fin de mejorar la eficiencia y rentabilidad de los procesos (Alimi, Rhif y Rebai, 2017; Beluli, 2019 Cho y Kim, 2019).

Sin embargo, el mercado actual impide la utilización máxima del recurso biomasa, ya que la concepción de la oferta y la demanda no es completa, no existen canales de comercialización abiertos y en muchos casos se crean dependencias o relaciones entre grupos de ofertantes y demandantes. Estas condiciones restringen la capacidad de negociación y precio de la biomasa (Cosentino et al., 2012), a esto se suma una tarifa máxima de venta energética por parte del ente estatal que establece las tarifas y todo ello restringe un precio de venta que sea rentable (Popescu, Mieila, Nica y Andrei, 2018).

\section{Perspectivas de mejora del mercado en el tiempo}

Si se incrementa la calidad de biomasa en el mercado, mediante el uso de biomasa homogénea, en conjunto con una mayor articulación entre los sectores productivos y una mejora de las políticas bioenergéticas, se puede producir el efecto de mejora explicado por (Beluli, 2019). Este autor establece que una mejoría en las condiciones de mercado afecta directamente la oferta y la demanda, incrementando el dinamismo y la competencia del sector tanto a lo interno (mejora de precios de biomasa, estabilidad en la demanda y posibilidades de negociación), como a lo externo (mejor precio de venta energético, permitiendo con ello competir con energías tradicionales o no renovables); además de un incremento del interés de los inversionistas, lo cual implicaría apertura de nuevos nichos de mercado, encadenamientos, economía circular, incentivos de inversión y expansión de la oferta y demanda. Estos aspectos se notaron con los resultados de la tabla 4 , en que la percepción de mejora en la calidad de la biomasa incidiría en crecimiento del mercado.

\section{CONCLUSIONES}

Se determinó que el mercado de la biomasa lignocelulósica de Costa Rica cuenta con una concordancia en cuanto a aspectos ambientales y sociales. Respecto a los niveles financiero y técnico, se tiene una diferencia en cuanto a la variabilidad de la biomasa. Hay una percepción homogénea por parte de los tres sectores del mercado de biomasa el cual es: complejo, dinámico, competitivo, modemo y carente de organización. Las condiciones percibidas son óptimas para el crecimiento y desarrollo de un mercado 
sostenible en el tiempo. Los demandantes mencionaron que las mejoras de la materia prima y de las políticas a escala de organización afectarían favorablemente la demanda en el tiempo y, con ello, la generación de nuevas fuentes de empleo e inversión.

\section{REFERENCIAS}

Alimi, M., Rhif, A., \& Rebai, A. (2017). Nonlinear dynamic of the renewable energy cycle transition in Tunisia: Evidence from smooth transition autoregressive models. International Journal of Hydrogen Energy, 42(13), 8670-8679. doi: 10.1016/j.ijhydene.2016.07.131

Baul, T., Datta, D., \& Alam, A. (2018). A comparative study on household level energy consumption and related emissions from renewable (biomass) and non-renewable energy sources in Bangladesh. Energy Policy, 114, 598-608, doi: 10.1016/j.enpol.2017.12.037

Beluli, V. (2019). Smart beer production as a possibility for cyber-attack within the industrial process in automatic control. Procedia Computer Science, 158, 206-213, doi: 10.1016/j.procs.2019.09.043

Bulut, U., \& Muratoglu, G. (2019). Renewable energy in Turkey: Great potential, low but increasing utilization, and an empirical analysis on renewable energy-growth nexus. Energy Policy, 123, 240-250, doi: 10.1016/j.enpol.2018.08.057

Cambero, C., Alexandre, M., \& Sowlati, T. (2015). Life cycle greenhouse gas analysis of bioenergy generation alternatives using forest and wood residues in remote locations: A case study in British Columbia, Canada. Resources, Conservation and Recyclin, 105(A), 5972, doi: 10.1016/j.resconrec.2015.10.014

Chidanand, F. C., Sisodia, G., \& Gopalan, S. (2019). A critical review on the utilization of storage and demand response for the implementation of renewable energy microgrids. Sustainable Cities and Society, 40, 735-745, doi: 10.1016/j.scs.2018.04.008

Cho, J., \& Kim, J. (2019). Multi-site and multi-period optimization model for strategic planning of a renewable hydrogen energy network from biomass waste and energy crops. Energy, 185, 527-40, doi: 10.1016/j.energy.2019.07.053

Cosentino, V., Favuzza, S., Graditi, G., Ippolito, M., Massaro, F., Sanseverino, E., \& Zizz, G. (2012). Smart renewable generation for an islanded system. Technical and economic issues of future scenarios. Energy, 39(1), 196-204. doi: 10.1016/j.energy.2012.01.030
Farhar, B. (1998). Gender and renewable energy: Policy, analysis, and market implications. Renewable Energy, 15(1-4), 230-239, doi: 10.1016/S0960-1481(98)00164-5

Fortini, B., \& Dye, K. (2017). At a global scale, do climate change threatened species also face a greater number of non-climatic threats? Global Ecology and Conservation, 11, 207-212, doi: 10.1016/j.gecco.2017.06.006

Franklin-Johnson, E. F., \& Canning, L. (2016). Resource duration as a managerial indicator for Circular Economy performance. Journal of Cleaner Production, 133, 589-598, doi, 10.1016/j.jclepro.2016.05.023

Gadaleta, M., Pellicciari, M., \& Berselli, G. (2019). Optimization of the energy consumption of industrial robots for automatic code generation. Robotics and Computer-Integrated Manufacturing, 57, 452464. doi: 10.1016/j.rcim.2018.12.020

Gazijahani, F., \& Salehi, J. (2018). Reliability constrained two-stage optimization of multiple renewable-based microgrids incorporating critical energy peak pricing demand response program using robust optimization approach. Energy,161, 9991015, doi: 10.1016/j.energy.2018.07.191

Hodges, G., Chapagain, B., Watcharaanantapong, D., Poudyal, N., Kline, K., \& Dale, V. (2019). Opportunities and attitudes of private forest landowners in supplying woody biomass for renewable energy. Renewable and Sustainable Energy Reviews, 113, doi: 10.1016/j.rser.2019.06.012

Instituto Costarricense de Electricidad [ICE] (2015). Potencialidad de nuevas ernergías de producción eléctrica. San José, Costa Rica: ICE.

Kahrl, F., Su, Y., Tennigkeit, T., Yang, Y., \& Xu, J. (2013). Large or small? Rethinking China's forest bioenergy policies. Biomass and Bioenergy, 59, 84-91, doi: 10.1016/j.biombioe.2012.01.042

Koengkan, M., Fuinhas, J., \& Marques, A. (2019). The effect of fiscal and financial incentive policies for renewable energy on CO2 emissions: the case for the Latin American region. Amsterdam: Academic Press.

Lee, L., \& Yang, J. (2019). Global energy transitions and political systems. Renewable and Sustainable Energy Reviews, 115, doi: 10.1016/j.rser.2019.109370

Lingcheng, K., Zhenning, Z., Jiaping, X., Jing, L., \& Yuping, C. (2019). Multilateral agreement contract optimization of renewable energy power grid-connecting under uncertain supply and market demand. Computers \& Industrial Engineering, 135, 689-701, doi: 10.1016/j.cie.2019.06.016

Mahidin, E., Mamat, M., Sani, M., Khoerunnisa, F., \& Kadarohman, A. (2019). Target and demand for renewable energy across 10 ASEAN countries by 2040. The Electricity Journal, 32(10),8-20, doi: 10.1016/j.tej.2019.106670 
Manolis, N., Zagas, T., Karetsos, G., \& Poravou, C. (2019). Ecological restrictions in forest biomass extraction for a sustainable renewable energy production. Renewable and Sustainable Energy Reviewss, 110, 290-297, doi: 10.1016/j.rser.2019.04.078.

Morseletto, P. (2020). Targets for a circular economy. Resources, Conservation and Recycling, 153,5-15 doi, 10.1016/j.resconrec.2019.104553.

Nematollahi, O., Hoghooghi, H., Rasti, M., \& Sedaghat, A. (2016). Energy demands and renewable energy resources in the Middle Eas. Renewable and Sustainable Energy Reviews, 54, 1172-1181

Pang, X., Mörtberg, U., Sallnäs, O., Trubins, R., Nordström, E., \& Böttcher, H. (2017). Habitat network assessment of forest bioenergy options using the landscape simulator LandSim - A case study of Kronoberg, southern Sweden. Ecological Modelling, 345, 99-112, doi: 10.1016/j.ecolmodel.2016.12.006

Popescu, G., Mieila, M., Nica, E., \& Andrei, J. (2018). The emergence of the effects and determinants of the energy paradigm changes on European Union economy. Renewable and Sustainable Energy Reviews, 81, 768-774, Doi: 10.1016/j.rser.2017.08.055

Röder, M., Thiffault, E., Martínez-Alonso, C., Senez-Gagnon, F., Paradis, L., \& Thornley, P. (2019). Understanding the timing and variation of greenhouse gas emissions of forest bioenergy systems. Biomass and Bioenergy, 121, 99-114, doi: 10.1016/j.biombioe.2018.12.019

Royston, S., Selby, J., \& Shove, E. (2018). Invisible energy policies: A new agenda for energy demand reduction. Energy Policy, 123, $127-$ 135.,doi: 10.1016/j.enpol.2018.08.052

Simangunsong, B., Sitanggang, V., Manurung, E., Rahmadi, A., Moore, G., Aye, L., \& Tambunan, A. (2017). Potential forest biomass resource as feedstock for bioenergy and its economic value in Indonesia. Forest Policy and Economics, 81, 10-17, doi: 10.1016/j.forpol.2017.03.022

Specht, J., \& Madlener, R. (2019). Energy Supplier 2.0: A conceptual business model for energy suppliers aggregating flexible distributed assets and policy issues raised. Energy Policy, 135,1-12, doi 10.1016/j.enpol.2019.110911

Ssempiira, J., Kissa, J., Nambuusi, B., Mukooyo, E., Opigo, J., Makumbi, F., . . ., \& Vounatsou, P. (2018). Interactions between climatic changes and intervention effects on malaria spatio-temporal dynamics in Uganda. Parasite Epidemiology and Control, 3(3), 1-11, doi: 10.1016/j.parepi.2018.e00070
Tiwary, A., Spasova, S., \& Williams, I. (2019). A community-scale hybrid energy system integrating biomass for localised solid waste and renewable energy solution: Evaluations in UK and Bulgaria. Renewable Energy, 39,960-967, doi: 10.1016/j.renene.2019.02.129

Valverde, J. C., Arias, D., Campos, R., Jiménez, M. F., \& Brenes, L. (2020). Forest and agro-industrial residues and bioeconomy: perception of use in the energy market in Costa Rica. Energy Ecology and Environment, 5(5),1-12, doi: 10.1007/s40974-02000172-4

Verlie, B. (2019). "Climatic-affective atmospheres": A conceptual tool for affective scholarship in a changing climate. Emotion, Space and Society, 33, 1-12, doi: 10.1016/j.emospa.2019.100623

Viviescas, C., Lima, L., Diuana, D., Vasquez, E., Ludovique, C., Silva, G., . . , \& Paredes, J. (2019). Contribution of Variable Renewable Energy to increase energy security in Latin America: Complementarity and climate change impacts on wind and solar resources. Renewable and Sustainable Energy Reviews, 113, doi: 10.1016/j.rser.2019.06.039

Zheng, Y., Jenkins, B., Kornbluth, K., Kendall, A., \& Træholt, C. (2018). Optimization of a biomass-integrated renewable energy microgrid with demand side management under uncertainty. Applied Energy, 230,836-844, doi: 10.1016/j.apenergy.2018.09.015

Manuscrito recibido el 12 de noviembre de 2019

Aceptado el 13 de abril de 2020

Publicado el 10 de diciembre de 2020

Este documento se debe citar como:

Valverde, J. C., Arias, D., Campos, R., Jiménez, M. F., \& Brenes, L. (2020). Análisis perceptual del mercado energético basado en biomasa lignocelulósica de origen arbóreo en Costa Rica. Madera y Bosques, 26(3), e2632066. doi: 10.21829/myb.2020.2362066

Madera y Bosques por Instituto de Ecología, A.C. se distribuye bajo una Licencia Creative Commons Atribución-NoComercialCompartirlgual 4.0 Internacional. 